\title{
Analysis of regulatory element evolution between human and mouse reveals a lack of cis-trans compensation
}

Kaia Mattioli ${ }^{1,2}$, Winona Oliveros ${ }^{3}$, Chiara Gerhardinger ${ }^{1}$, Daniel Andergassen ${ }^{1}$, Philipp G. Maass ${ }^{4,5}$, John L. Rinn ${ }^{6}$, Marta Melé, ${ }^{+, 3}$

${ }^{\dagger}$ corresponding author

${ }^{1}$ Department of Stem Cell and Regenerative Biology, Harvard University, Cambridge, MA, 02138, USA

${ }^{2}$ Department of Biological and Biomedical Sciences, Harvard Medical School, Boston, MA, 02115, USA

${ }^{3}$ Life Sciences Department, Barcelona Supercomputing Center, Barcelona, Catalonia, 08034, Spain

${ }^{4}$ Genetics and Genome Biology Program, Sickkids Research Institute, Toronto ON, M5G 0A4, Canada

${ }^{5}$ Department of Molecular Genetics, University of Toronto, Toronto, ON, M5S 1A1, Canada

${ }^{6}$ Department of Biochemistry, University of Colorado, BioFrontiers Institute, Boulder, CO, 80301, USA

\section{ABSTRACT}

1 Gene expression differences between species are driven by both cis and trans effects. Whereas cis

2 effects are caused by genetic variants in close proximity to the target gene, trans effects are due to distal genetic variants that affect diffusible elements such as transcription factors. Previous studies have mostly assessed the impact of cis and trans effects at the gene level. However, how cis and trans effects differentially impact regulatory elements such as enhancers and promoters remains poorly understood. Here, we used massively parallel reporter assays to directly measure cis and trans effects between human and mouse embryonic stem cells at thousands of individual regulatory elements. Our approach revealed that cis effects are widespread across regulatory elements, and the strongest cis effects are associated with the disruption of motifs recognized by strong transcriptional activators. Conversely, we found that trans effects are rare but stronger in enhancers than promoters, and can be attributed to a subset of transcription factors that are differentially expressed between human and mouse. While previous studies have found extensive co-occurrence of cis and trans effects in opposite directions that stabilize gene expression throughout evolution, we find that cis-trans compensation is uncommon within individual regulatory elements. Thus, our results are consistent with a model wherein compensatory cistrans effects at the gene level are explained by cis and trans effects that separately impact several regulatory elements rather than cis-trans effects that occur simultaneously within a single regulatory element. Together, these results indicate that studying the evolution of individual regulatory elements is pivotal to understand the tempo and mode of gene expression evolution. 


\section{INTRODUCTION}

Since King and Wilson suggested that changes in transcriptional regulation underlie phenotypic differences between species ${ }^{1}$, it has become clear that changes in gene expression are heritable and often play a role in the evolution of phenotypes ${ }^{2,3}$. Changes in non-coding regulatory elements-including promoters and enhancers-are particularly important in driving the evolution of gene expression ${ }^{4,5}$. Two primary mechanisms are responsible for the evolution of gene expression: cis effects and trans effects. Cis effects are due to genetic variants that are in linkage disequilibrium with the target gene; for example, genetic variants located in gene promoters or enhancers that affect transcription factor (TF) binding sites. Conversely, trans effects are driven by diffusible elements (such as TFs) that are distal and unlinked to the genes they affect. Any given gene can be subject to cis effects, trans effects, or both ${ }^{6}$.

Much work has assessed the contribution of cis and trans effects on the evolution of gene expression. One of the most common approaches has been to perform allele-specific RNA sequencing of F1 hybrid offspring, which can separate regulatory variants acting in cis (which show allele-specific effects in the hybrid) from those acting in trans (which affect both hybrid alleles equally) ${ }^{6}$. These studies have assessed both intra- and inter-species variation in gene expression across a variety of taxa, including yeast $^{7,8}$, insects ${ }^{9,10}$, plants ${ }^{11}$, and mice ${ }^{12}$. These and other studies have shown a predominance of cis effects, but highlighted a role for trans effects that varied across taxa. Moreover, cis and trans effects were found to often occur simultaneously and affect target gene expression in opposite directions ${ }^{12}$. This so-called "compensation" between cis and trans effects is thought to stabilize gene expression throughout evolution $^{6}$. A major limitation of these studies, however, is that while they can assign cis and trans effects to target genes, they cannot disentangle effects at individual regulatory elements. Studies on regulatory element evolution have found that the number of regulatory elements-especially enhancers-that target a gene influence the tempo and mode of gene expression evolution ${ }^{5,13}$. However, only small scale studies have examined how cis and trans effects drive differences in regulatory element activities across species $^{14,15}$.

The development of massively parallel reporter assays (MPRAs) has revolutionized our ability to dissect the regulatory element code ${ }^{16,17}$. Indeed, MPRAs have been used to measure regulatory element activity of thousands of sequences across tissues ${ }^{18}$, species $^{15}$, and allelic variants ${ }^{18-20}$. In this work, we use MPRAs to quantitatively investigate cis and trans effects across thousands of individual regulatory elements including enhancers, promoters of protein-coding genes, and promoters of long non-coding RNA (IncRNA) genes. We perform MPRAs in similar cellular environments from two mammalian species_embryonic stem cells (ESCs) from human and mouse - to perform a systematic analysis of cis and trans effects at thousands of individual regulatory elements simultaneously. 
bioRxiv preprint doi: https://doi.org/10.1101/847491; this version posted November 20, 2019. The copyright holder for this preprint (which was not certified by peer review) is the author/funder, who has granted bioRxiv a license to display the preprint in perpetuity. It is made available under aCC-BY-NC-ND 4.0 International license.

\section{RESULTS}

\section{Designing an MPRA to measure regulatory element evolution}

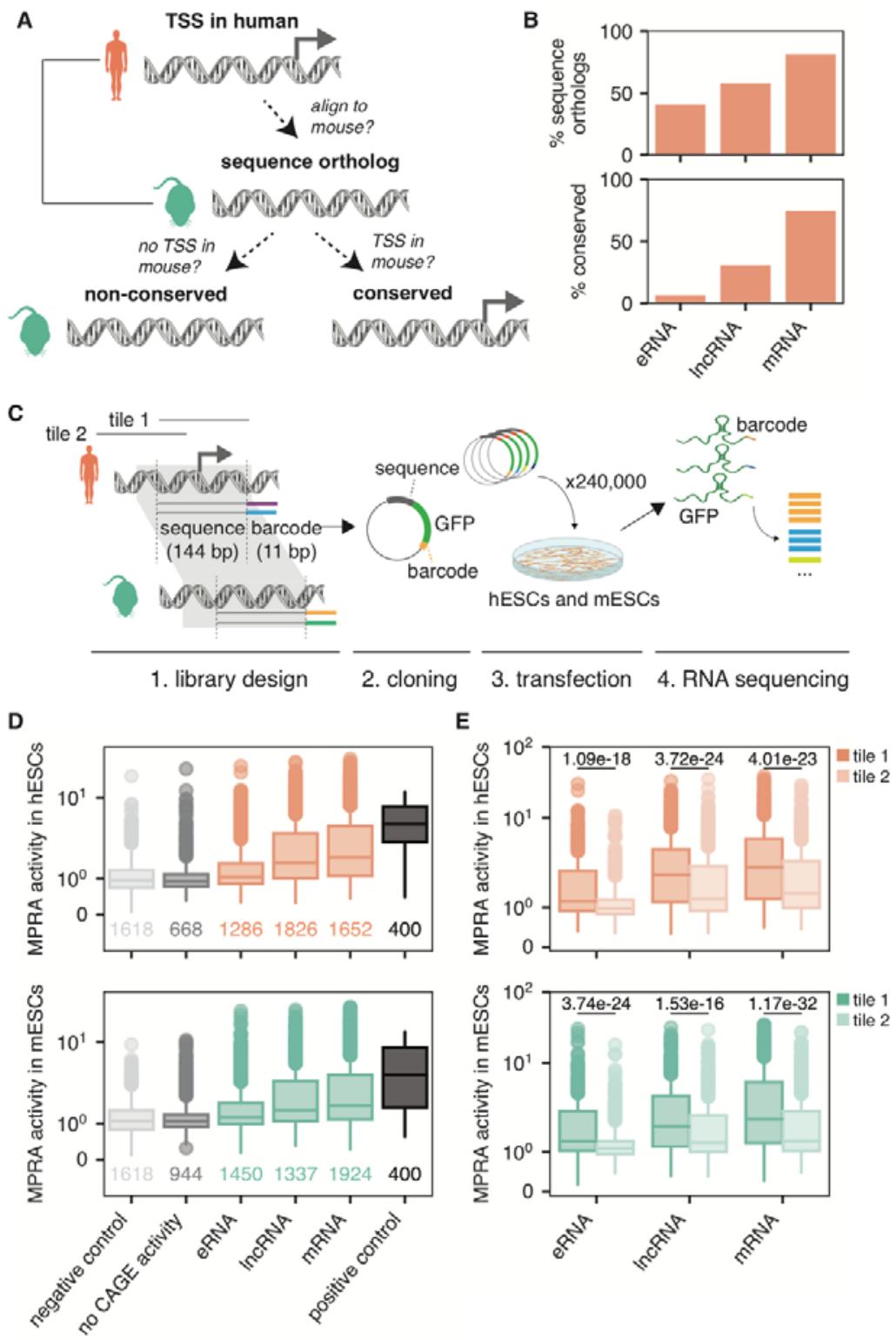

Figure 1: MPRA in human and mouse ESCs recapitulates endogenous gene expression patterns A: Schematic depicting the definitions of a sequence ortholog and conserved/non-conserved TSSs. Sequence orthologs are TSS regions whose sequences can be reciprocally mapped to the other species. Conserved TSSs are a subset of sequence orthologs that also overlap a TSS in the other species (defined as having $\geq 10$ CAGE reads in $\geq$ 1 sample), whereas non-conserved TSSs do not.

B: Percentage of human-to-mouse sequence orthologs and conserved TSSs broken up by biotype.

C: Schematic of MPRA design. Tile 1 overlaps the assigned TSS (114 bp upstream to 30 bp downstream) whereas tile 2 does not (228 bp upstream to 84 bp upstream).

D: MPRA activities of human (top) and mouse (bottom) TSSs in their native contexts, human ESCs and mouse ESCs, respectively, broken up by whether they have endogenous CAGE activity and then by biotype and compared to negative controls (random sequences) and positive controls (CMV promoter regions).

E: MPRA activities of TSS-overlapping tile (tile 1) compared to upstream tile (tile 2) across all human biotypes (top) and mouse biotypes (bottom). P-value shown is from a two-sided Mann Whitney test. 
To investigate regulatory element evolution between human and mouse, we first defined regulatory elements in both species using a set of robust transcription start sites (TSSs) defined by the FANTOM5 consortium ${ }^{21}$. We categorized these TSSs into three major biotypes: (1) eRNAs (RNAs emerging from bidirectionally transcribed enhancers that do not overlap protein-coding genes), (2) IncRNA promoters, and (3) mRNA promoters (see methods). We then projected these TSSs onto the genome of the other species (i.e., human TSSs were projected onto the mouse genome and vice versa). We classified TSSs as "sequence orthologs" if we were able to reciprocally map the TSS between the two species. We further classified the "sequence ortholog" TSSs as conserved TSSs if the aligned region in the other species (+/- 50 bp from the TSS) contained evidence of an active TSS (Figure 1A; see methods). As expected, the proportion of TSS that were sequence orthologs and conserved were both highest in mRNAs and lowest in eRNAs (Figure 1B; Supplemental Figure S1). Despite moderate levels of sequence orthology in eRNAs and IncRNAs, both biotypes exhibited very high activity turnover, with only $7 \%$ and $31 \%$ of human eRNA TSSs and IncRNA TSSs being conserved in mouse, respectively.

To systematically assess the contribution of cis and trans effects to the evolution of thousands of regulatory elements simultaneously, we performed a massively parallel reporter assay (MPRA) (Figure 1C). MPRAs measure the transcriptional activities of designed sequences in a cell type of interest, and thus enable us to test both cis effects (how do orthologous sequences compare within a given cellular environment) and trans effects (how do different cellular environments affect a given sequence). As early development is known to play a key role in evolutionary processes ${ }^{22}$, we chose to perform the MPRA in a developmentally-relevant cell type: human and mouse ESCs. Thus, we selected 3,327 pairs of orthologous regulatory elements between human and mouse, all of which had endogenous activity in either human or mouse ESCs or both (Supplemental Figure S2; Supplemental Table S1; see methods). The full list of regulatory elements in our library can be found in Supplemental Table S2. To ensure that we covered all regulatory activity sequence surrounding the TSS, we designed two oligonucleotide tiles for each TSS (Figure 1C). All told, our library included 13,533 sequences to test (Supplemental Table S3). To control for technical variation across sequencing measurements, each element was represented a minimum of 13 times, each time with a different barcode. We also included randomly-generated sequences as negative controls (with 3 barcodes each) as well as tiled regions of the cytomegalovirus (CMV) promoter as positive controls (with 60 barcodes each), resulting in a final library of 181,065 unique oligonucleotides (Supplemental Table S4). We performed three biological replicates each in human ESCs (hESC) and mouse ESCs (mESCs) and confirmed that replicates of hESCs and mESCs clustered separately (Supplemental Figure S3 and Supplemental Figure S4). We then removed barcodes with low counts, resulting in a set of 2,952 regulatory sequence pairs that were well represented in our data (see methods).

We next quantified each sequence's ability to drive transcription in the MPRA experimenttermed "MPRA activity"- using MPRAnalyze ${ }^{23}$. Briefly, MPRAnalyze uses a graphical model to estimate the rate of transcription of each sequence in the library by comparing RNA counts for each barcode to 
100 input DNA counts for each barcode. To determine whether our MPRA was able to recapitulate biological

101 signal, we compared the MPRA activity of each regulatory element in its native context (human

102 sequences in hESCs and mouse sequences in mESCs) to negative and positive control sequences (see

103 methods). TSSs were more active than negative controls and mimicked endogenous activity levels:

104 eRNAs had the lowest activity while mRNAs had the highest activity (Figure 1D).

105 We then compared the activity of the annotated TSS-overlapping tiles (tile 1) to the upstream tiles

106 (tile 2) (Figure 1C). As expected, across all biotypes, annotated TSS-overlapping tiles were significantly

107 more active in their native context than the upstream tiles (Figure 1E). In 18\% of regulatory element

108 pairs, however, the upstream tile was more active than the TSS-overlapping tile in both species'

109 (Supplemental Figure S5), likely due to slight misannotation of the exact TSS location. Thus, while

110 FANTOM5-defined TSSs are highly accurate, including additional upstream regions in the MPRA can

111 help to refine core promoter locations. We therefore assigned each of the 2,952 regulatory element pairs

112 a single representative tile to use in both species: we always used the annotated TSS-overlapping tile

113 except in those cases where the upstream tile had more activity in both species. Among those, 1,644

114 pairs $(55 \%)$ had significant MPRA activity (MPRA q-value $<0.05$ ) in at least 1 native context. We limited

115 all of our subsequent analyses to this set of 1,644 active sequence pairs (3,288 sequences total). 


\section{Cis effects are common and associated with evolutionary turnover}
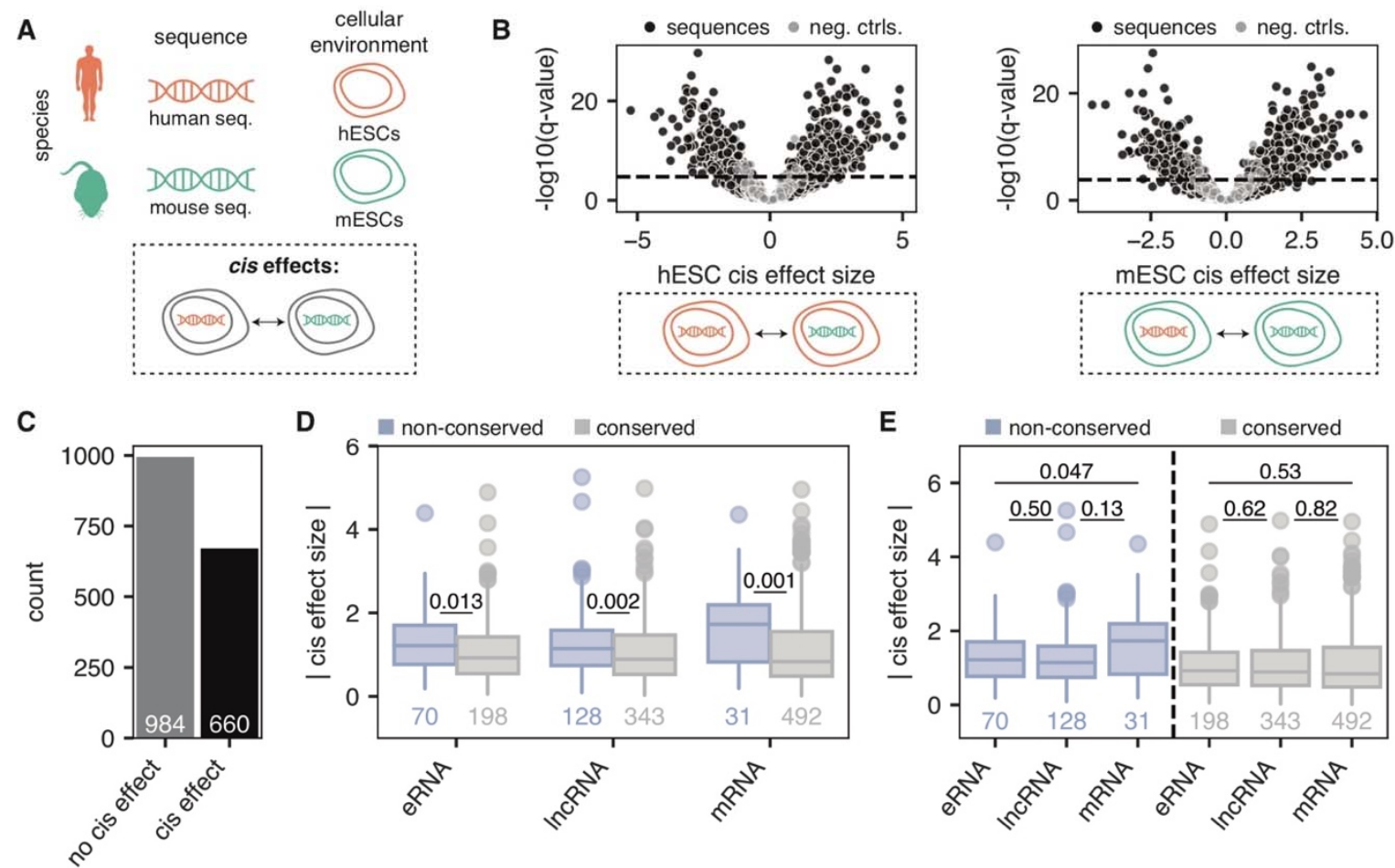

Figure 2: $40 \%$ of orthologous regulatory elements show significant cis effects

116

117

118

119

120

121

122

123

124

125

126

127

128

129

130

131

132

133

134

135

136

137

A: Schematic depicting the definition of a cis effect: MPRA activity differences between human sequences and mouse sequences while keeping the cellular environment constant.

B: Volcano plot showing the cis effect sizes (log2 foldchanges in activity between sequences in hESCs, left and mESCs, right) of orthologous sequences (black) and null differential controls (gray). Horizontal line depicts an empirical FDR cut-off of 0.1 , calculated using null differential controls (see methods).

C: Count of orthologous sequence pairs with significant cis effects.

D: Absolute cis effect sizes across biotypes, broken up into non-conserved TSSs (blue) and conserved TSSs (gray).

P-values shown are from a two-sided Mann Whitney test.

E: Same as (D), but this time comparing differences across biotypes. P-values shown are from a two-sided Mann Whitney test.

Differences in regulatory element activity between species could be due to differences in DNA sequence (cis effects) or cellular context differences (trans effects) between the species or both. We decided to focus first on cis effects, which can be attributed to differences in DNA sequence alone. We defined cis effects as the MPRA activity differences between orthologous sequence pairs in the same cellular environment (Figure 2A). To calculate cis effects, we used MPRAnalyze to test for MPRA activity differences between pairs of orthologous regulatory elements. An advantage of using MPRAnalyze is that it is able to use information from null differential controls to inform its comparative model. The ideal null differential controls are pairs of identical sequences tagged with different barcodes. We therefore leveraged our CMV tiles, each of which was attached to 60 barcodes, to create our null differential controls by down-sampling barcodes (Supplemental Figure S6 and Supplemental Figure S7; see methods). As expected, orthologous regulatory element pairs had higher cis effect sizes than null differential controls in both hESCs and mESCs (Figure 2B). Overall, $40 \%$ of the 1,644 tested regulatory 
138 element pairs showed a significant cis effect in hESCs, mESCs, or both (empirical FDR $<0.1$ ) (Figure

139 2C; see methods).

We next sought to examine how cis effects differ across biotypes, including conserved and non-

141 conserved TSSs. Within each biotype, non-conserved regulatory element pairs showed significantly

142 higher cis effects than conserved regulatory element pairs (Figure 2D). We confirmed that our non-

143 conserved pairs are bona fide non-conserved regulatory elements, as the non-conserved pairs we had

144 defined (non-conserved TSSs and their orthologous sequence in the other species that lacked a TSS)

145 had higher pairwise alignment scores than they did to the closest TSS in the other species

146 (Supplemental Figure S8). Thus, these non-conserved regulatory elements are not due to

147 misalignments between genomes. Cis effect sizes across biotypes were relatively uniform (Figure 2E).

148 However, non-conserved mRNA TSSs showed the highest cis effect sizes (Figure 2E), consistent with

149 the idea that the largest jump in activity is from mRNA TSSs - which have the highest activity out of all

150 biotypes-to sequences without a TSS at all. 


\section{Cis effects are associated with disruption of certain TF motifs}

A

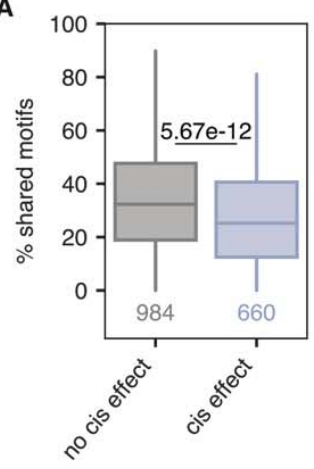

D

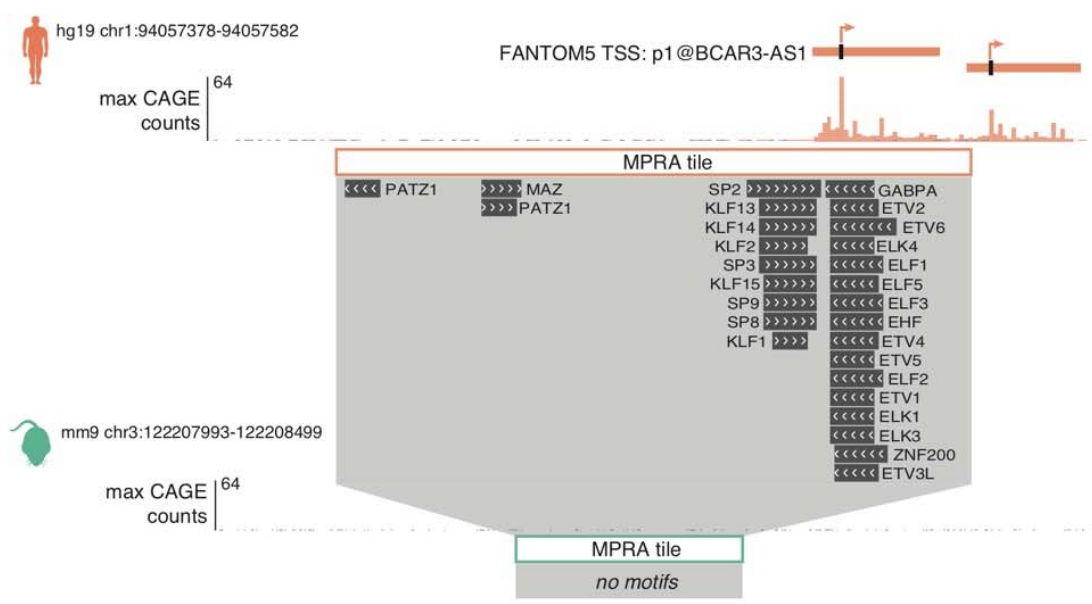

B
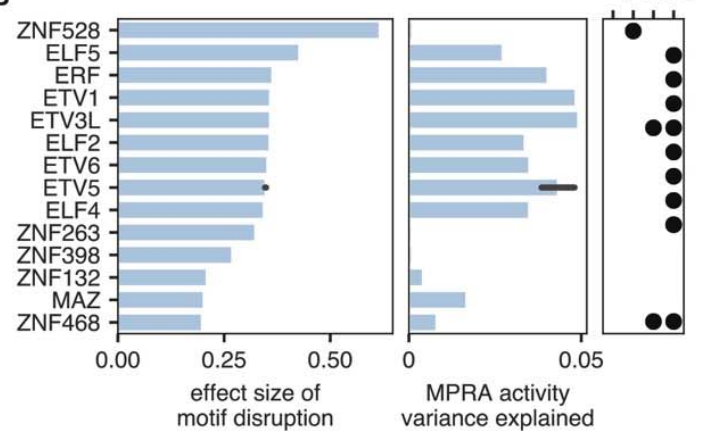

variance explained

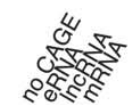

C

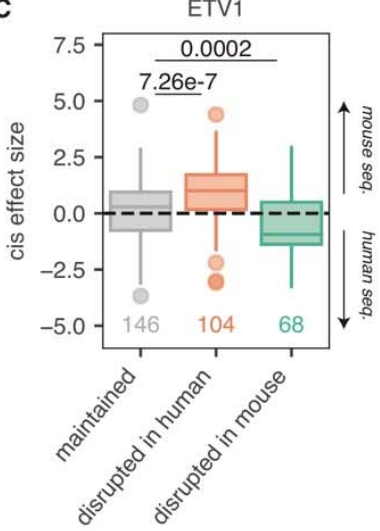

E

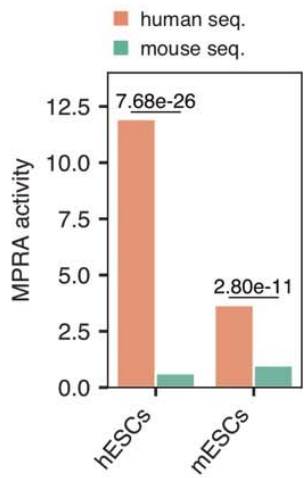

Figure 3: Disruption of certain motifs is associated with cis effects

A: Percentage of shared motifs in tiles that show cis effects vs. those that do not. P-value shown is from a two-sided Mann Whitney test.

B: Plot showing the activating motifs whose disruption is significantly associated with cis effects $(F D R<0.05)$. Left: effect size associated with motif disruption. Middle: additional variance in MPRA activity explained by the TF. Right: enrichment of a given TF motif across biotypes, as determined by a Hypergeometric test. Black dots denote significant enrichment (FDR < 0.05). The ETV5 TF has two "best" motifs according to the curated Lambert et al. TF list and therefore the average of these two motifs are plotted, with the bootstrapped $95 \%$ confidence interval shown. C: Relationship between cis effect sizes and the ETV1 motif, where "maintained" are sequence pairs that both have the ETV1 motif, "disrupted in human" are pairs where the ETV1 motif is present in mouse but not in human, and "disrupted in mouse" are pairs where the ETV1 is present in human but not in mouse. A cis effect size $>0$ indicates the mouse sequence has higher activity whereas a cis effect size $<0$ indicates the human sequence has higher activity. P-values shown are from a two-sided Mann Whitney test.

D: Genome browser screenshot of an example locus showing a cis effect. Only motifs that were found to explain $\geq 1 \%$ of the variance in MPRA activity are shown.

E: MPRA activities for human sequence (orange) and mouse sequence (green) in hESCs and mESCs for the locus shown in D. P-values shown are the q-values calculated by MPRAnalyze.

Cis effects are often caused by disruption of motifs that are recognized by sequence-specific transcription factors (TFs). Thus, we next sought to determine the relationship between cis effects and TF motifs. Previous work showed that only a subset of TF motifs can be reliably associated with MPRA activity variance ${ }^{24}$. Thus, we selected a set of 466 motifs from TFs that are expressed in hESCs and 
mESCs and are associated with MPRA activity (Supplemental Figure S9; Supplemental Table S5) either as activators or as repressors for further analysis. As expected, regulatory element pairs showing no cis effects shared more TF motifs than sequence pairs with significant cis effects (Figure 3A), reinforcing the notion that the more TF motifs two sequences have in common, the more similar their activity levels. In addition, we found 17 individual motifs were significantly associated with cis effects when disrupted. The majority of these motifs were predicted activators and enriched in mRNAs (Figure 3B); indeed, several of the strongest effect sizes could be attributed to the ETS transcription factors, including the oncogenic TF ETV1 ${ }^{25}$ (Figure 3C). However, we also found a subset of motifs that were predicted repressors and enriched in eRNAs (Supplemental Figure S10). Thus, while cis effects can generally be attributed to the disruption of strong activating motifs, in rarer cases, cis effects are due to the disruption of weak repressive motifs. While this may reflect real biological effects, it may also be due to the fact that MPRAs are more powered to detect activators over repressors ${ }^{24,26}$.

An example of a cis effect can be seen at the TSS for the human-specific IncRNA BCAR3-AS1. In human, the strongest core promoter region of BCAR3-AS1 contains many strong activating motifs, including several ETS motifs (Figure 3D). The orthologous region in the mouse, however, shows no CAGE activity and lacks these motifs, as there is no orthologous IncRNA in the mouse (Figure 3D). As expected, the pairwise alignment score between the human BCAR3-AS1 TSS and region and the region shown in Figure 3D is higher than the alignment score to the nearest mouse TSS (160.9 compared to 138.5), indicating that our MPRA tiles are correctly aligned. In our MPRA, this pair shows a significant cis effect: the human sequence is significantly more active than the orthologous mouse sequence in both hESCs and mESCs (Figure 3E). Collectively, our results show that cis effects are common-especially in regulatory element pairs that show activity changes between species-and associated with disruption of specific TF motifs. 


\section{Trans effects are rare and highest in eRNAs}

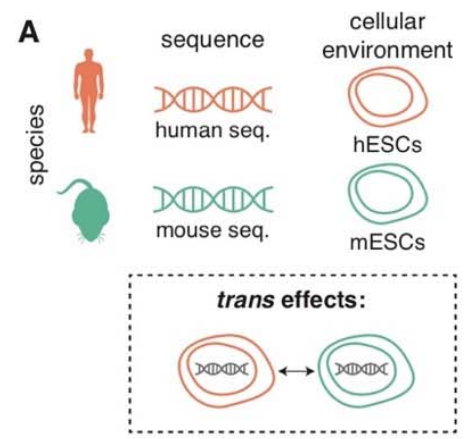

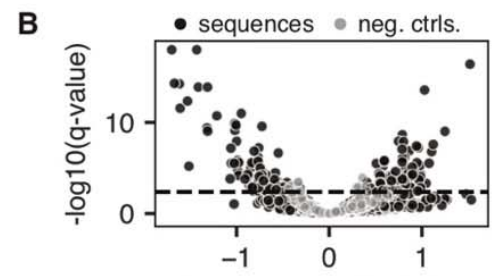

human trans effect size

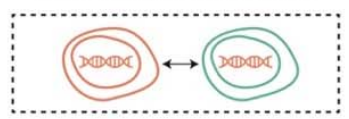

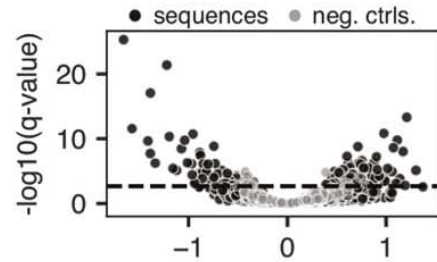

mouse trans effect size

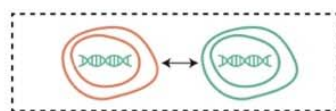

C

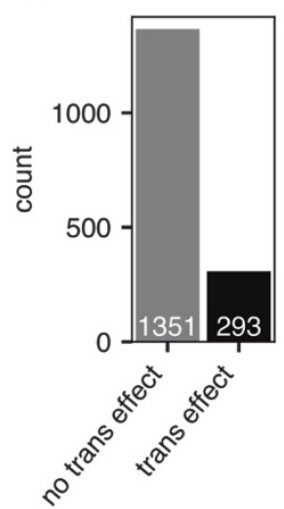

D
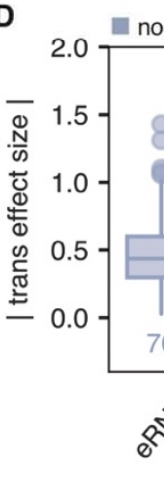

non-conserved conserved

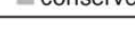

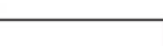

E

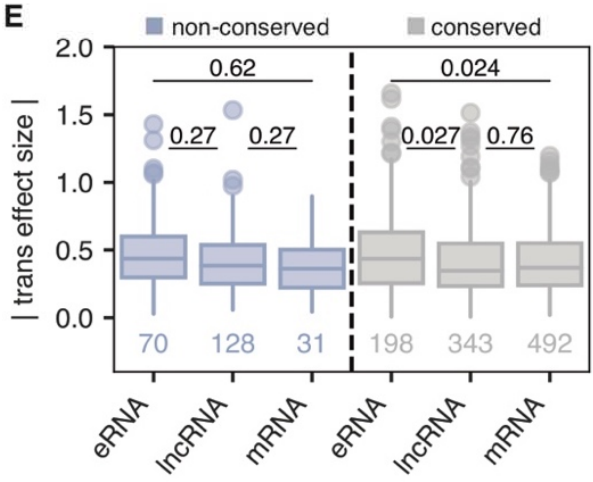

Figure 4: $18 \%$ of orthologous regulatory elements show significant trans effects

A: Schematic depicting the definition of a trans effect: MPRA activity differences between hESCs and mESCs while keeping the sequence constant.

B: Volcano plot showing the trans effect sizes (log2 foldchanges in cell type for human sequences, let and mouse sequences, right) of regulatory sequences (black) and null differential controls (gray). Horizontal line depicts an empirical FDR cut-off of 0.1 , calculated using null differential controls (see methods).

C: Count of orthologous sequence pairs with significant trans effects.

D: Absolute trans effect sizes across biotypes, broken up into non-conserved TSSs (blue) and conserved TSSs (gray). P-values shown are from a two-sided Mann Whitney test.

E: Same as (D), but this time comparing differences across biotypes. P-values shown are from a two-sided Mann Whitney test.

After quantifying cis effects, we next sought to quantify trans effects. We defined trans effects as the difference in MPRA activity driven by differences in cellular environment alone and measured them by quantifying MPRA activity differences between hESCs and mESCs while keeping the sequence constant (Figure 4A). As with cis effects, human and mouse regulatory elements showed higher trans effects than null differential controls (Figure 4B). Overall, 18\% of the 1,617 filtered regulatory element pairs with significant activity showed a significant trans effect in the human sequence, the mouse sequence, or both (Figure 4C). Compared to cis effect sizes, however, trans effect sizes were much lower. In addition, unlike cis effects, we found that within each biotype, trans effects were similar between conserved and not conserved TSSs (Figure 4D). While trans effect sizes were low in general, we found that conserved eRNA TSSs had the highest trans effect sizes (Figure 4E). We speculate that this may reflect the fact 
bioRxiv preprint doi: https://doi.org/10.1101/847491; this version posted November 20, 2019. The copyright holder for this preprint (which was not certified by peer review) is the author/funder, who has granted bioRxiv a license to display the preprint in perpetuity. It is made available under aCC-BY-NC-ND 4.0 International license.

214 that enhancers are often redundant-i.e., multiple enhancers regulate the same target gene-and this 215 may allow for them to absort trans effects at minimal fitness costs. 
bioRxiv preprint doi: https://doi.org/10.1101/847491; this version posted November 20, 2019. The copyright holder for this preprint (which was not certified by peer review) is the author/funder, who has granted bioRxiv a license to display the preprint in perpetuity. It is made available under aCC-BY-NC-ND 4.0 International license.

\section{A subset of differentially-expressed TFs are associated with trans effects}

A

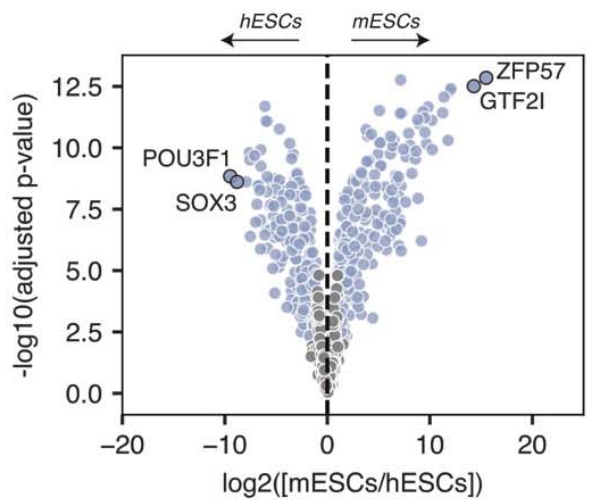

C

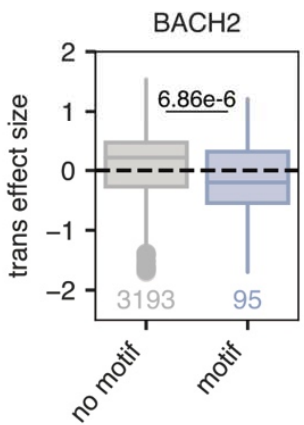

D

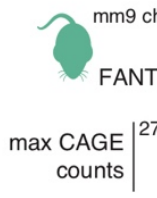

\section{so}

B

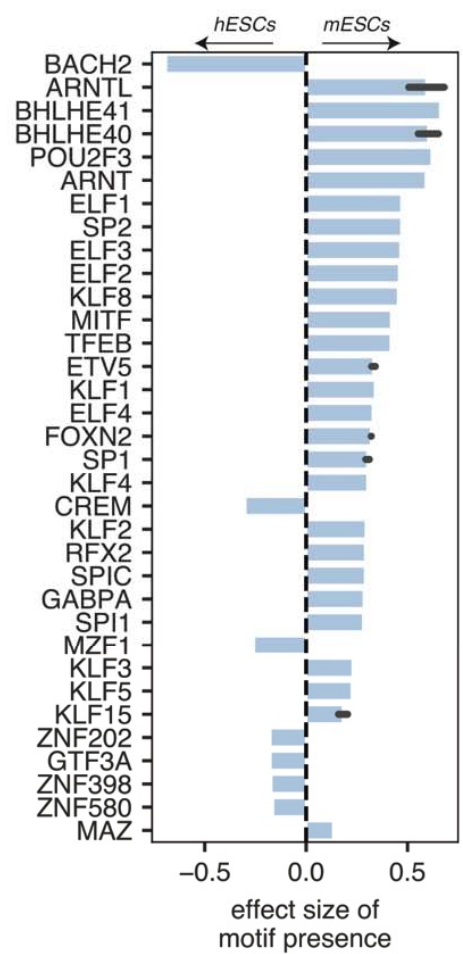

motif presence
hESCS MESCS

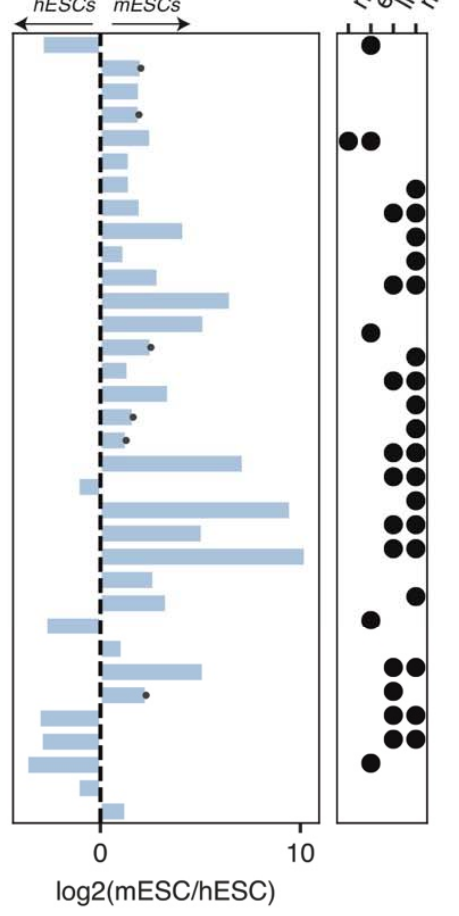

POU2F3

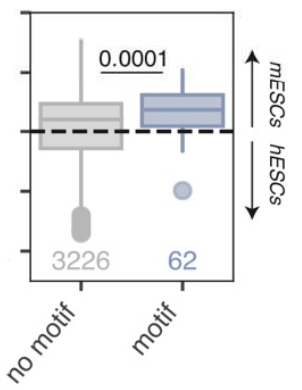

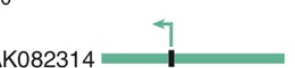

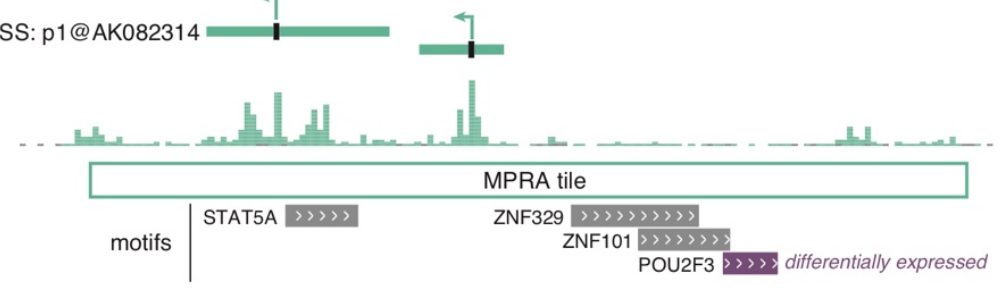

E

mESCs

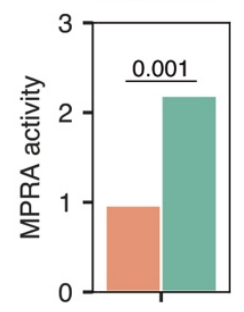

Figure 5: Trans effects are associated with a subset of differentially-expressed TFs

A: Volcano plot showing the differential expression of orthologous human and mouse TFs in hESCs and mESCs. most differentially expressed TFs in either direction are highlighted.

B: Plot showing the activating motifs significantly associated with trans effects (FDR < 0.05$)$ that are also differentially expressed between hESCs and mESCs in the expected direction. Left: effect size associated with motif enrichment. Motifs that are associated with sequences more highly expressed in mESCs are $>0$, and those associated with sequences more highly expressed in hESCs are < 0 . Middle: log2 foldchange in expression via RNA-seq. Right: enrichment of a given TF motif across biotypes, as determined by a Hypergeometric test. Black dots denote significant enrichment (FDR <0.05).

C: Relationship between trans effect sizes and the BACH2 and POU2F3 motifs. Trans effect sizes for sequences with motif and without motif. Trans effect sizes $>0$ indicate higher activity in mESCs while effect sizes $<0$ indicate higher activity in mESCs. P-values shown are from two-sided Mann Whitney tests.

D: Genome browser screenshot of an example locus showing a trans effect. Gray motifs correspond to TFs that are not differentially expressed between hESCs and mESCS; the purple motif, POU2F3, is differentially expressed. E: MPRA activities in hESCs (orange) and mESCs (green) for the mouse locus shown in D. P-value shown is the FDR value calculated by MPRAnalyze. 
We next focused on identifying the TFs associated with the observed trans effects. We used a linear model to determine whether motif presence was significantly associated with trans effect sizes (see methods). After adjusting for multiple hypothesis testing, we found that 137 TFs (corresponding to 156 unique motifs) were significantly associated with trans effects. As motifs for different TFs can often be very similar to each other ${ }^{27}$ (e.g., all POU TFs share the consensus motif ATGCAAAT), we reasoned that while we found many motifs to be significantly associated with trans effects, only a subset of these TFs were likely driving the trans effect signal. To hone in on these, we performed RNA sequencing to determine differential expression of TFs between our hESCs and mESCs (see methods). We limited our analysis to the 1,032 TFs known to be one-to-one orthologs between human and mouse. Of these TFs, 661 of these TFs were expressed in either hESCs or mESCs and 428 were significantly differentially expressed (absolute log2 fold-change $\geq 1$ and FDR < 0.01) between hESCs and mESCs (Figure 5A;

\section{Supplemental Table S6). Of the 120 TFs we found to be significantly associated with trans effects, 67} were one-to-one orthologs differentially expressed between hESCs and mESCs (Supplemental Figure S11). We reasoned that TFs likely driving trans effects would match in the direction of their differential expression and the direction of their trans effects. Of the 67 aforementioned TFs, 44 (66\%) agreed in the directionality of their differential expression and trans effect enrichment (Figure 5B). These included both constitutively-active TFs (e.g. SP1, ARNT) as well as tissue-specific TFs (e.g. immune factor BACH2, developmental regulator POU2F3/OCT11) (Figure 5C).

An example of a trans effect can be seen at the promoter of the uncharacterized mouse IncRNA AK082314 (Figure 3D). This region harbors 4 motifs for 4 TFs: STAT5A, ZNF329, ZNF101, and POU2F3. Of these 4 TFs, the only one that is differentially expressed between hESCs and mESCs is POU2F3, which is expressed $\sim 5$-fold more highly in mESCs than hESCs. Consistent with this, in our MPRA, the AK082314 promoter shows significantly higher activity in mESCs than in hESCs. Collectively, our results show that we can pinpoint a subset of TFs that may be driving trans effects between hESCs and mESCs. 
bioRxiv preprint doi: https://doi.org/10.1101/847491; this version posted November 20, 2019. The copyright holder for this preprint (which was not certified by peer review) is the author/funder, who has granted bioRxiv a license to display the preprint in perpetuity. It is made available under aCC-BY-NC-ND 4.0 International license.

\section{Co-occurrence of cis and trans effects in opposite directions are rare at individual regulatory elements}
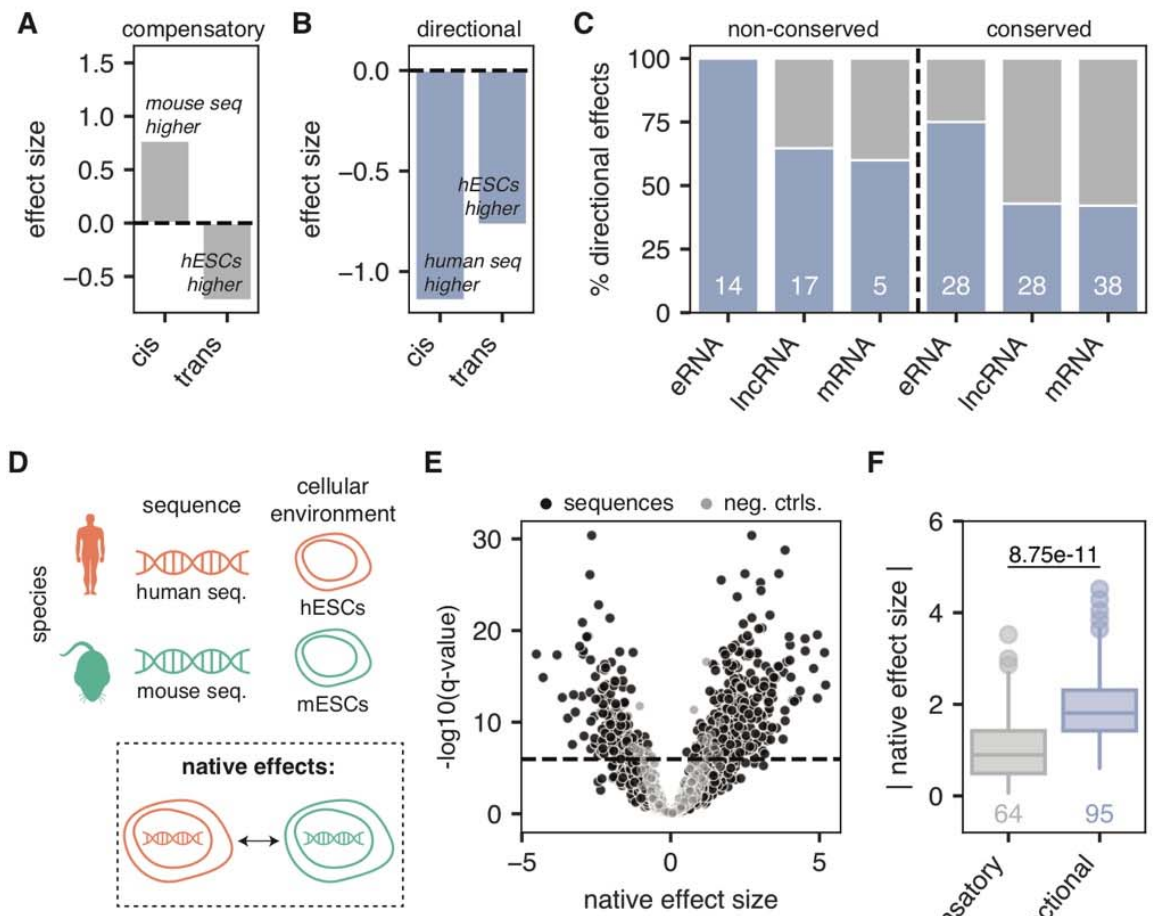

E

F

G

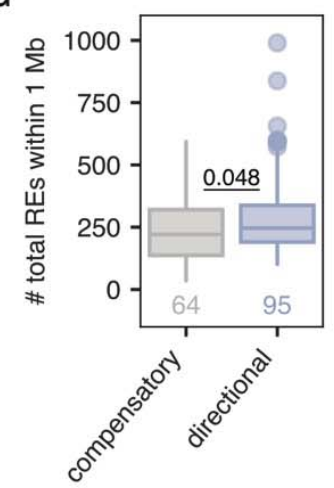

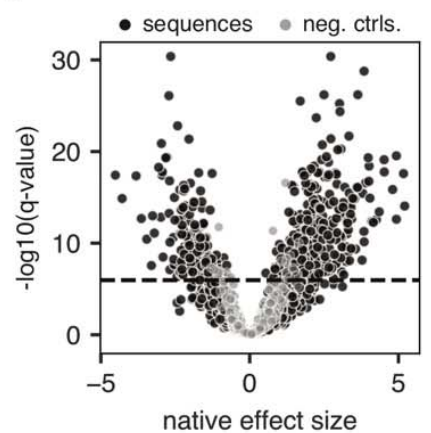

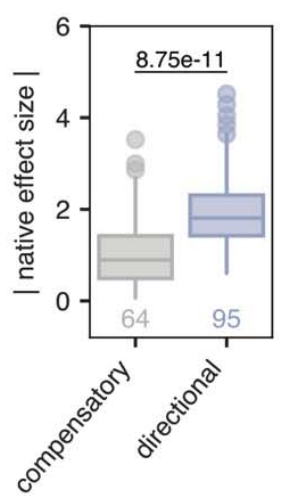

H

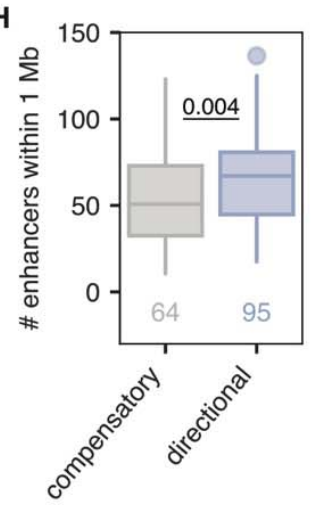

I

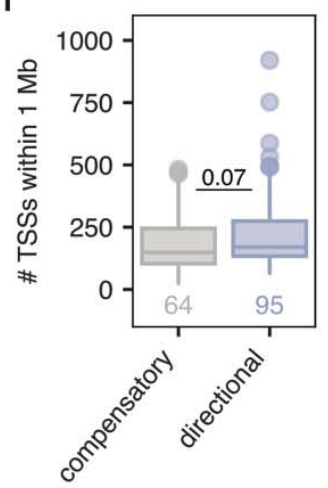

Figure 6: Only $40 \%$ of regulatory pairs show evidence of compensation between cis and trans effects A-B: Example of a compensatory cis-trans effect (A) and a directional cis-trans effect (B). Effect sizes $>0$ indicate higher activity in the mouse sequence or cellular environment whereas effect sizes $<0$ indicate higher activity in the human sequence or cellular environment.

C: Percent of regulatory element pairs across biotypes with directional cis/trans effects. Only pairs with both cis and trans effects are considered and the number in each group are shown.

D: Schematic showing overview of how native effects are defined

E: Volcano plot of native effect sizes for orthologous regulatory element pairs (black) compared to null differential controls (gray). Horizontal line depicts an empirical FDR cut-off of 0.1 , calculated using null differential controls (see methods).

F: Absolute native effect sizes for sequences showing compensatory cis-trans effects compared to directional cistrans effects. P-value shown is from a two-sided Mann Whitney test.

G-I: Number of additional FANTOM5 regulatory elements within a $1 \mathrm{Mb}$ window of a given regulatory element, broken up by whether they show compensatory cis-trans effects or directional cis-trans effects. P-values shown are from a two-sided Mann Whitney test. G: total regulatory elements (enhancers and promoters), H: enhancers only, I:

promoters only. 
Cis and trans effects can co-occur, and previous gene-based studies have shown an excess of cis and trans effects occurring in opposite directions ${ }^{6,12}$. These so-called "compensatory" cis-trans effects help to stabilize gene expression over evolutionary time. It is unclear, however, whether the observed compensation between cis and trans effects occurs at the individual regulatory element level, or whether the compensation occurs primarily across different regulatory elements that regulate the same target gene ${ }^{4}$. We therefore sought to examine the extent of cis-trans compensation occurring within individual regulatory elements.

Of the 794 regulatory element pairs with either a cis or a trans effect, we found that $159(20 \%)$ showed both cis and trans effects (odds $=2.01, p=8.6 \times 10^{-8}$, Fisher's exact test). We then determined how often the co-occurrence of cis and trans effects was compensatory (i.e., the two effects were in opposite directions - for example, Figure 6A depicts a regulatory element pair with a cis effect showing that the mouse sequence is more active, but a trans effect showing that the human environment results in higher activity) or "directional" (i.e., the two effects were in the same direction-for example, Figure 6B depicts a regulatory element pair where the cis and trans effects are both higher for the human sequence and cell type, respectively). Surprisingly, we found that the majority $(60 \%, p=0.017$, binomial test) of cistrans co-occurrences were directional. This was driven by an excess of directional effects at nonconserved TSSs-especially eRNAs (Figure 6C). Conversely, conserved IncRNA and mRNA TSSs showed mostly compensatory effects. Thus, whereas regulatory element turnover between human and mouse is associated with directional cis-trans effects, regulatory element conservation is associated with cis-trans compensation.

We next wondered whether the regulatory elements that show cis-trans compensation show evidence of stabilized activity between species. To this end, we examined the activity of regulatory element pairs in their native environments-human sequences in hESCs and mouse sequences in mESCs. We reasoned that if compensatory cis-trans effects stabilize regulatory element activity, we should see that regulatory elements in their native environments show virtually equal MPRA activities. To this end, we quantified said "native effects" between regulatory element pairs (Figure 6D-E). Indeed, regulatory element pairs showing compensatory cis-trans effects showed very low differences in native activity, whereas regulatory element pairs showing directional cis-trans effects showed large differences in native activity (Figure 6F). Thus, quantitative regulatory element activity levels are stabilized and destabilized by compensatory and directional cis-trans effects, respectively.

Recent work has shown that genes regulated by larger numbers of regulatory elements tend to have more stable transcription throughout evolution ${ }^{13}$. We therefore hypothesized that perhaps regulatory elements lacking redundancy (i.e., having fewer "partner" regulatory elements that regulate the same target gene) may show more evidence of cis-trans compensation than regulatory elements with high redundancy, which show more inter-element compensation. To test this, for each regulatory element, we counted the number of additional FANTOM5 regulatory elements (both TSSs and enhancers) that lied within a 1 megabase window surrounding it. We found that less redundant regulatory elements have 
308 more cis-trans compensation than highly redundant regulatory elements (Figure 6G). Moreover, this

309 effect was driven by the number of nearby enhancers (Figure $6 \mathrm{H}$ ) and not by the number of nearby

310 promoters (Figure 6I). Thus, regulatory elements surrounded by few nearby enhancers tend to show

311 compensatory cis-trans effects. Collectively, our results support a model whereby compensation between

312 cis and trans effects within an individual regulatory element is more likely to occur at less redundant

313 regulatory elements, perhaps because in these regions, there is less opportunity for inter-element

314 compensation. 


\section{DISCUSSION}

In this work, we sought to characterize the mode underlying the evolution of individual regulatory elements that are orthologous between human and mouse by focusing on enhancers, IncRNA promoters, and mRNA promoters. Overall, we find that trans effects are less common and generally weaker than cis effects across all regulatory elements. These results are consistent with the prevailing model where cis effects preferentially accumulate between species, likely because trans effects result in more deleterious pleiotropic side-effects that are selected against ${ }^{6}$. We also see interesting differences between biotypes. While cis effect sizes are generally uniform across conserved eRNAs, IncRNAs, and mRNAs (Figure 2E), trans effects are highest in conserved eRNAs (Figure 4E). This suggests that the evolutionary trajectory of conserved IncRNAs is more similar to that of conserved mRNAs, whereas eRNAs behave as a separate group. Indeed, the larger accumulation of trans effects in eRNAs is likely due to their higher redundancy. Finally, the high resolution of our assay allowed us to identify 44 TFs that are associated with trans effects. Future work aimed at understanding species-level differences between human and mouse ESCs could use this set of 44 TFs as a starting point.

Previous studies have found that when cis and trans effects co-occur at the same gene, they are more often compensatory (i.e., act in different directions) than directional (i.e., act in the same direction) ${ }^{6,12}$, and are driven by stabilizing selection on transcript levels. However, when assessing cistrans contributions at regulatory elements rather than genes, we do not find evidence of an excess of cistrans compensation. Indeed, we only find evidence of enrichment for cis-trans compensation at conserved IncRNA and mRNA TSSs but not at eRNAs or non-conserved TSSs (Figure 6C). Similarly, a recent publication showed that, when looking at TF binding across different mouse strains, cis-trans compensation was only enriched in conserved TF binding sites ${ }^{28}$. Thus, while natural selection acts to stabilize the functional product of the gene-the transcript level一the evolutionary trajectories of individual regulatory elements are not as straightforward. Indeed, recent work has shown that ensembles of highly redundant enhancers are often poorly conserved, despite stable expression of their target gene throughout evolution ${ }^{13}$. Such data is supportive of a model wherein regulatory elements can undergo evolutionary flux and compensate for one another over time. Along these lines, here we find that highly redundant regulatory elements are less likely to show compensatory cis-trans effects than regulatory elements acting in smaller numbers (Figure 6H). Collectively, our data are consistent with the idea of inter-enhancer compensation. We propose that when regulatory elements are highly redundant, interenhancer compensation between cis and trans effects dominates, but when regulatory elements are less redundant, compensation between cis and trans effects can occur at the individual regulatory element level (Figure 7). 
gene regulated by highly redundant enhancers
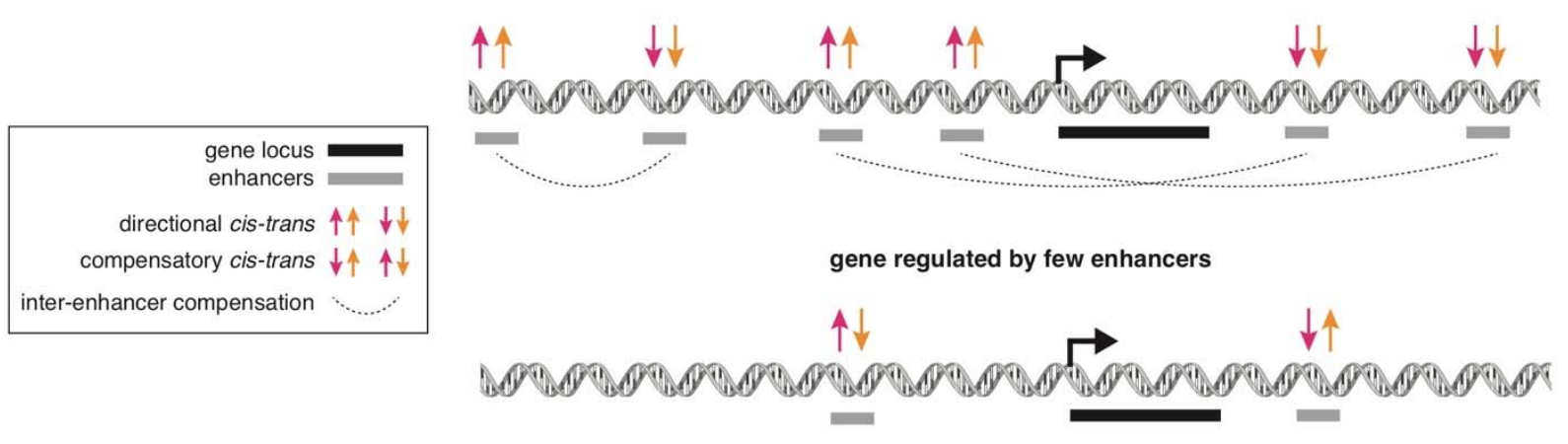

Figure 7: Model of inter- vs. intra- enhancer compensation.

Top: at a gene regulated by many redundant enhancers, enhancers are free to show directional cis-trans effects because there is ample opportunity for crosstalk between enhancers, leading to inter-enhancer compensation. Bottom: at a gene regulated by very few enhancers, individual enhancers show compensatory cis-trans effects (intraenhancer) because there is less opportunity for crosstalk between enhancers.

In this study we sought to perform an unbiased assessment of cis and trans effects between human and mouse across a variety of biotypes. To this end, we leveraged MPRAs to systematically test the contribution of cis and trans effects to the evolution of thousands of regulatory elements. While the use of MPRAs is extremely powerful, it also has some limitations. For example, we could only study a subset of all existing regulatory elements in the human and mouse genomes. However, the sequences that we tested were carefully selected in an unbiased manner so that they would be representative of regulatory elements genome-wide. Another limitation of our approach is that we only assessed two species in one cellular background (ESCs). Although gene expression between hESCs and mESCs is similar in general, distinct differences between the two cell lines exist ${ }^{29}$. Moreover, whether the known differences between hESCs and mESCs are reflective of differences in isolation and culture conditions ${ }^{30}$ or underlying species-specific biology remains controversial ${ }^{31}$. Future work is needed to assess whether similar patterns exist in other tissues, and the extent to which these patterns may affect fundamental biological processes in a species-specific manner. Nevertheless, our work has characterized the baseline to which information from other tissues and other species can be added in order to gain a more complete picture of the evolution of regulatory elements.

In summary, we find that the mode of evolution can differ at different classes of regulatory elements. Notably, we find that compensation between cis and trans effects at individual regulatory elements is rare, supporting the idea that compensation across regulatory elements-rather than within individual regulatory elements-is a widespread feature of mammalian genomes ${ }^{13}$. Future work should focus on integrating the mode of evolution at individual regulatory elements with target transcript expression levels across species. Collectively, our results underscore the importance of examining the role of individual regulatory elements in the evolution of gene expression. 


\section{METHODS}

\section{TSS selection and biotype assignment}

To assign accurate TSSs to genes, we intersected human and mouse GENCODE genes ${ }^{32}$ ( $v 19$ in human and vM13 in mouse) with FANTOM5 TSSs ${ }^{21}$ in both species. Specifically, we found the closest FANTOM5 TSS (on the same strand) within +/- 1000 bp of the GENCODE-annotated TSS. We classified any gene having a GENCODE gene_type of "protein_coding" as an mRNA. We classified any gene included in the GENCODE long_noncoding_RNAs gtf file as a IncRNA, provided it showed no evidence of a conserved open reading frame (PhyloCSF ${ }^{33}$ ORF score $<0$ and branch length $<0.1$ ). We classified any FANTOM5-annotated "robust" enhancers ${ }^{34}$ as eRNAs, and used both the sense and antisense TSS provided by FANTOM5. More details are available in the Supplemental Methods (TSS selection and biotype assignment section).

\section{Sequence orthology assignment}

To determine sequence orthologs, we first mapped human TSSs (hg19) to mouse (mm9) and vice versa using the liftOver program with the parameter minMatch $=1$. We then reciprocally mapped the lifted-over TSSs back to their original species, and required that they map to the exact same original TSS nucleotide. As FANTOM5 enhancers have two TSSs, we required that both TSSs reciprocally map in order to consider an enhancer a sequence ortholog.

\section{Conserved TSS assignment}

To determine conserved vs. non-conserved TSSs, we intersected the lifted-over TSSs with the maximum CAGE read coverage in that species (ctssTotalCounts bigwig files downloaded from the FANTOM5 data hub $\left.^{35}\right)$. We determined a TSS to be conserved if the region immediately surrounding the TSS (+/-50 bp) contained $\geq 10$ maximum CAGE reads. As enhancers have two TSSs, if either of the TSSs intersected $\geq$ 10 maximum CAGE reads, we considered it conserved.

\section{MPRA sequence pair selection}

We required all sequence pairs in the MPRA library to have a either an annotated CAGE peak in human or mouse that is expressed above background in either hESCs or mESCs $(\geq 0.024$ normalized counts in $\mathrm{hESCs}$ and $\geq 0.022$ normalized counts in mESCs, Supplemental Figure S2). We included all IncRNAs (and their orthologous sequences) that met this threshold in the pool. We randomly selected the remaining biotypes in roughly equal numbers, given that they met this expression threshold. As eRNAs have two TSSs, we included both of its TSSs and both of its TSSs' orthologous sequences in the pool. Exact numbers of each biotype in the MPRA can be found in Supplemental Table S1 and the list of regulatory elements included in the MPRA can be found in Supplemental Table S2 and. More details can be found in the Supplemental Methods (MPRA sequence pair selection section).

\section{MPRA oligonucleotide design}

Each oligonucleotide we designed was 200 bp long, containing 144 bp of regulatory sequence, an 11 bp barcode, and $45 \mathrm{bp}$ of sequence necessary for cloning. For each TSS selected above, we included two $144 \mathrm{bp}$ tiles: one directly surrounding the TSS (-114/+30 bp) and one slightly upstream of the TSS (-228/$84 \mathrm{bp}$ ) (Supplemental Table S3). We then generated 1,622 random 144bp sequences to serve as negative controls. We also tiled across the CMV promoter in 144 bp segments to create 4 positive control tiles. We assigned TSS regions 13 barcodes, random sequences 3 barcodes, and CMV sequences 60 barcodes (Supplemental Table S4). More details can be found in the Supplemental Methods (MPRA oligonucleotide design section). 


\section{MPRA cloning, transfection, and sequencing}

Twist Bioscience synthesized the oligo pool, which we then cloned as previously described ${ }^{18}$ into plasmids to generate a library of constructs where the regulatory sequence is upstream of a reporter gene (here, GFP) that is upstream of a unique barcode. $\square$ We assayed the initial representation of barcodes using high-throughput DNA-sequencing. We transfected these constructs into live cells and performed three biological replicates each in hESCs (HUES64 cells) and mESCs (derived from mouse blastocysts ${ }^{36}$ ) corresponding to three consecutive passages (Supplemental Figure S3). We isolated RNA and assayed barcode expression by high-throughput RNA-sequencing. More details can be found in the Supplemental Methods (MPRA cloning, transfection, and sequencing section).

\section{MPRA Analysis}

All code to reproduce analyses is available at https://github.com/kmattioli/2019_cis_trans_MPRA.

\section{Quantifying MPRA activity}

After trimming and quality filtering DNA and RNA reads, we mapped exact matches to known barcodes and 10 upstream constant nucleotides of GFP. We only measured sequences that had at least $50 \%$ of their barcodes represented at $\geq 10$ counts in the input DNA library. We used the R package MPRAnalyze ${ }^{23}$ to quantify MPRA activities for each sequence in each condition using the program's "quantification" mode. We used our randomly-generated sequences as the background null distribution, as the majority of these sequences should not induce transcription. More details can be found in the Supplemental Methods (quantifying MPRA activities section).

\section{Calculating differential MPRA activity}

After quantifying MPRA activity and assigning 1 tile to each sequence pair (Supplemental Figure S5), we used MPRAnalyze $e^{23}$ to perform differential activity analyses using the program's "comparison" mode. In comparison mode, as the null hypothesis is not the lack of transcription but the lack of differential transcription, we used down-sampled barcodes corresponding to identical CMV sequences as the background null distribution. In each of the 5 models (cis effects in hESCs, cis effects in mESCs, trans effects of mouse sequences, trans effects of human sequences, and native effects), we tested whether the full model was a better fit than an intercept-only model using a likelihood ratio test. More details can be found in the Supplemental Methods (calculating differential MPRA activity section).

\section{Calling significant differential effects}

We considered sequences to have significant cis, trans, or native effects if the q-value calculated by MPRAnalyze was less than the q-value that resulted in $<10 \%$ of negative controls being called significant, which is effectively an empirical FDR of 0.1 (Supplemental Figure S6). We also required effect sizes to be higher than the minimum significant null differential control effect size (Supplemental Figure S7). We assigned each sequence pair one cis and trans effect size: we used the maximum cis or trans effect size between the two models (hESCs/mESCs for cis and human/mouse for trans) unless the effect was only significant in one model, in which case used the corresponding significant effect size. More details can be found in the Supplemental Methods (Calling significant differential effects section).

\section{Motif mapping}


We used a curated list of human TFs defined by Lambert et $\mathrm{al}^{27}$. We then used the CisBP ${ }^{38}$ positionweight matrices designated by Lambert et al to be the "best" motifs for each of these TFs. In total, this list contained 1360 motifs corresponding to 1104 unique TFs. We mapped these motifs in both human sequences and mouse sequences using the FIMO program from the MEME suite with default parameters $^{39}$.

\section{Finding motifs predictive of MPRA activity}

For each motif, we fit a linear model to mean MPRA activity across all sequences as follows:

$$
\text { mean }(M P R A \text { activity }) \sim G C \text { content }+C p G \text { content }+ \text { motif present }
$$

and determined whether the binary motif present indicator explained significantly more of the variance than a reduced model without the indicator using a likelihood ratio test (Supplemental Table S5). We used the Python statsmodels ${ }^{40}$ package to run all linear models. More details can be found in Supplemental Methods (Finding motifs predictive of MPRA activity section).

\section{Finding motifs associated with cis and trans effects}

For each motif, we fit a linear model to absolute cis effect sizes across all sequence pairs as follows:

$$
\mid \text { cis effect size }|\sim \operatorname{mean}(G C)+\operatorname{mean}(C p G)+| \Delta(G C)|+| \Delta(C p G) \mid+ \text { motif disrupted }
$$

and determined whether the motif disrupted parameter (indicating whether a motif was present in only one of the two paired sequences) was significant (FDR $<0.05$ ).

For each motif, we fit a linear model to trans effect sizes across all sequences as follows:

$$
\text { trans effect size } \sim G C+C p G+\text { motif present }
$$

and determined whether the motif present parameter was significant $(F D R<0.05)$. More details available in Supplemental Methods (Finding motifs associated with cis and trans effects sections).

\section{RNA-seq of hESCs and mESCs}

We sequenced both untransfected and transfected hESCs and mESCs. We extracted RNA from TRIzol using standard protocols and used the Illumina TruSeq kit (non-stranded) to create polyA+ libraries from total RNA. We measured library concentration using the Qubit dsDNA HS Assay kit (Thermo Fisher Scientific), and ran all of the libraries on a Bioanalyzer (Agilent) to assess purity and fragment size, and sequenced on a HiSeq 2500 at Harvard University's Bauer Sequencing Core (75 bp paired end).

\section{RNA-seq analysis}

We aligned reads to either hg19 or mm10 using Hisat $2^{41}$. We used FeatureCounts to count reads aligning to genes in either GENCODE v25 (human) or GENCODE vM13 (mouse) ${ }^{42}$. We downloaded orthologous genes between human and mouse from Ensembl (version 96) ${ }^{43}$, and removed any orthologs classified as "many-to-many". We quantified gene expression in each transfected sample using DESeq2 ${ }^{44}$. To find differentially expressed genes, we used the edgeR-limma pipeline ${ }^{45}$ (filtering out any genes with cpm $<1$ ) to model paired samples (transfected and untransfected) and control for transfection status.

\section{ACKNOWLEDGEMENTS}


476

477

478

479

480

We thank Veronika Akopian for help with HUES64 cell culture, Abigail Groff for providing mESCs, Jordan Lewandowski for help with mESC cell culture, and Martha Bulyk for helpful discussions. M.M. was a Gilead Fellow of the Life Sciences Research Foundation during part of the project. K.M. was a National Science Foundation Graduate Research Fellow under grant no. DGE1144152 during the majority of the project. J.L.R. is an HHMI faculty scholar.

\section{AUTHOR CONTRIBUTIONS}

481

482

483

484

485

\section{DATA ACCESS}

K.M. and M.M. designed the project and wrote the manuscript. K.M. designed the oligonucleotide libraries and performed all MPRA computational analyses. W.O. analyzed FANTOM5 CAGE data and performed RNA-seq analysis. C.G. and P.G.M. performed the MPRA experiments. C.G. performed RNA-seq. D.A. assisted with cell culture. J.L.R. contributed to the project design and discussion. All authors have read and approved the manuscript for publication.

The MPRA sequencing data and genomic RNA-seq data from this study have been submitted to the NCBI Gene Expression Omnibus (GEO; http://www.ncbi.nlm.nih.gov/geo/) under accession number GSE140574. All scripts required to reproduce this work are available on GitHub at https://github.com/kmattioli/2019_cis_trans_MPRA 


\section{REFERENCES}

1. King, M. C. \& Wilson, A. C. Evolution at two levels in humans and chimpanzees. Science 188, 107-16 (1975).

2. Stern, D. L. \& Orgogozo, V. THE LOCI OF EVOLUTION: HOW PREDICTABLE IS GENETIC EVOLUTION? Evolution (N. Y). 62, 2155-2177 (2008).

3. Romero, I. G., Ruvinsky, I. \& Gilad, Y. Comparative studies of gene expression and the evolution of gene regulation. Nat. Rev. Genet. 13, 505-516 (2012).

4. Danko, C. G. et al. Dynamic evolution of regulatory element ensembles in primate CD4+ T cells. Nat. Ecol. Evol. 2, 537-548 (2018).

5. Berthelot, C., Villar, D., Horvath, J. E., Odom, D. T. \& Flicek, P. Complexity and conservation of regulatory landscapes underlie evolutionary resilience of mammalian gene expression. Nat. Ecol. Evol. 2, 152-163 (2018).

6. Signor, S. A. \& Nuzhdin, S. V. The Evolution of Gene Expression in cis and trans. Trends Genet. 34, 532-544 (2018).

7. Emerson, J. J. et al. Natural selection on cis and trans regulation in yeasts. Genome Res. 20, 826-836 (2010).

8. Tirosh, I., Reikhav, S., Levy, A. A. \& Barkai, N. A Yeast Hybrid Provides Insight into the Evolution of Gene Expression Regulation. Science (80-. ). 324, 659-662 (2009).

9. Wittkopp, P. J., Haerum, B. K. \& Clark, A. G. Regulatory changes underlying expression differences within and between Drosophila species. Nat. Genet. 40, 346-350 (2008).

10. Osada, N., Miyagi, R. \& Takahashi, A. Cis- and Trans-regulatory Effects on Gene Expression in a Natural Population of Drosophila melanogaster. Genetics 206, 2139-2148 (2017).

11. Shi, X. et al. Cis- and trans-regulatory divergence between progenitor species determines geneexpression novelty in Arabidopsis allopolyploids. Nat. Commun. 3, 950 (2012).

12. Goncalves, A. et al. Extensive compensatory cis-trans regulation in the evolution of mouse gene expression. Genome Res. 22, 2376-84 (2012).

13. Danko, C. G. et al. Dynamic evolution of regulatory element ensembles in primate CD4+ T cells. Nat. Ecol. Evol. 2, 537-548 (2018).

14. Gordon, K. L. \& Ruvinsky, I. Tempo and Mode in Evolution of Transcriptional Regulation. PLoS Genet. 8, e1002432 (2012).

15. Ryu, H. et al. Massively parallel dissection of human accelerated regions in human and chimpanzee neural progenitors. bioRxiv 256313 (2018). doi:10.1101/256313

16. Patwardhan, R. P. et al. Massively parallel functional dissection of mammalian enhancers in vivo. Nat. Biotechnol. 30, 265-270 (2012).

17. Melnikov, A. et al. Systematic dissection and optimization of inducible enhancers in human cells using a massively parallel reporter assay. Nat. Biotechnol. 30, 271-277 (2012).

18. Mattioli, K. et al. High-throughput functional analysis of IncRNA core promoters elucidates rules governing tissue specificity. Genome Res. (2019). doi:10.1101/gr.242222.118

19. Ulirsch, J. C. et al. Systematic Functional Dissection of Common Genetic Variation Affecting Red Blood Cell Traits. Cell 165, 1530-1545 (2016).

20. Tewhey, R. et al. Direct Identification of Hundreds of Expression-Modulating Variants using a Multiplexed Reporter Assay. Cell 165, 1519-1529 (2016).

21. Forrest, A. R. R. et al. A promoter-level mammalian expression atlas. Nature 507, 462-70 (2014).

22. Hall, B. K. Evolutionary Developmental Biology (Evo-Devo): Past, Present, and Future. Evol. Educ. Outreach 5, 184-193 (2012).

23. Ashuach, T. et al. MPRAnalyze: statistical framework for massively parallel reporter assays. Genome Biol. 20, (2019).

24. Ernst, J. et al. Genome-scale high-resolution mapping of activating and repressive nucleotides in regulatory regions. Nat. Biotechnol. 34, 1180-1190 (2016).

25. Jané-Valbuena, J. et al. An Oncogenic Role for ETV1 in Melanoma. Cancer Res. 70, 2075-2084 (2010).

26. Movva, R. et al. Deciphering regulatory DNA sequences and noncoding genetic variants using neural network models of massively parallel reporter assays. PLoS One 14, e0218073 (2019).

27. Lambert, S. A. et al. The Human Transcription Factors. Cell 172, 650-665 (2018).

28. Wong, E. S. et al. Interplay of cis and trans mechanisms driving transcription factor binding and 
gene expression evolution. Nat. Commun. 8, (2017).

29. Ginis, I. et al. Differences between human and mouse embryonic stem cells. Dev. Biol. 269, 360380 (2004).

30. Tesar, P. J. et al. New cell lines from mouse epiblast share defining features with human embryonic stem cells. Nature 448, 196-199 (2007).

31. Takahashi, S., Kobayashi, S. \& Hiratani, I. Epigenetic differences between naïve and primed pluripotent stem cells. Cell. Mol. Life Sci. 75, 1191-1203 (2018).

32. Frankish, A. et al. GENCODE reference annotation for the human and mouse genomes. Nucleic Acids Res. 47, D766-D773 (2019).

33. Lin, M. F., Jungreis, I. \& Kellis, M. PhyloCSF: a comparative genomics method to distinguish protein coding and non-coding regions. Bioinformatics 27, i275-82 (2011).

34. Andersson, R. et al. An atlas of active enhancers across human cell types and tissues. Nature 507, 455-461 (2014).

35. Lizio, M. et al. Gateways to the FANTOM5 promoter level mammalian expression atlas. Genome Biol. 16, 22 (2015).

36. Groff, A. F., Barutcu, A. R., Lewandowski, J. P. \& Rinn, J. L. Enhancers in the Peril lincRNA locus regulate distant but not local genes. Genome Biol. 19, 219 (2018).

37. Gifford, C. A. et al. Transcriptional and Epigenetic Dynamics during Specification of Human Embryonic Stem Cells. (2013). doi:10.1016/j.cell.2013.04.037

38. Weirauch, M. T. et al. Determination and Inference of Eukaryotic Transcription Factor Sequence Specificity. Cell 158, 1431-1443 (2014).

39. Grant, C. E., Bailey, T. L. \& Noble, W. S. FIMO: scanning for occurrences of a given motif. Bioinformatics 27, 1017-1018 (2011).

40. Seabold, S. \& Perktold, J. Statsmodels: Econometric and Statistical Modeling with Python. PROC. OF THE 9th PYTHON IN SCIENCE CONF (2010).

41. Kim, D., Langmead, B. \& Salzberg, S. L. HISAT: A fast spliced aligner with low memory requirements. Nat. Methods 12, 357-360 (2015).

42. Liao, Y., Smyth, G. K. \& Shi, W. featureCounts: an efficient general purpose program for assigning sequence reads to genomic features. Bioinformatics 30, 923-930 (2014).

43. Aken, B. L. et al. Ensembl 2017. Nucleic Acids Res. 45, D635-D642 (2017).

44. Love, M. I., Huber, W. \& Anders, S. Moderated estimation of fold change and dispersion for RNAseq data with DESeq2. Genome Biol. 15, (2014).

45. Robinson, M. D., McCarthy, D. J. \& Smyth, G. K. edgeR: a Bioconductor package for differential expression analysis of digital gene expression data. Bioinformatics 26, 139-140 (2010). 